\title{
Barriers to and enablers of kangaroo mother care
}

Soon Min Lee, MD

Department of Pediatrics, Gangnam Severance Hospital, Yonsei University College of Medicine, Seoul, Korea

Kangaroo mother care (KMC) is defined as early, continuous, and prolonged skin-to-skin contact between the mother and preterm baby, exclusive breastfeeding, early discharge after hospital-initiated KMC with continuation at home, and adequate support and follow-up for mothers at home. ${ }^{1)}$ The World Health Organization issued recommendations for the care of preterm infants, including KMC, in 2015. ${ }^{1)}$ Globally, the Every Newborn Action Plan emphasizes KMC as an essential component of neonatal health initiatives. ${ }^{2)}$

KMC has been identified as a useful and effective intervention for improving the outcomes of preterm infants. Compared with conventional care, KMC reduced mortality by $40 \%$, sepsis by $65 \%$, and hypothermia by $72 \% .{ }^{3)}$ It also reduced the incidence of severe illness and lower respiratory tract disease, hypoglycemia, and hospital readmissions. ${ }^{3)}$ Moreover, KMC increased weight, length, head circumference, breastfeeding, maternal satisfaction, and maternal-infant attachment. ${ }^{3,4)}$ It also led to beneficial neurodevelopmental, behavioral, social, cognitive, and emotional outcomes. ${ }^{5}$ However, limited national adoption and implementation of KMC was observed, and its global coverage remains low. $\left.{ }^{6}\right)$ In addition, there are significant variations in the onset and duration of skin-to-skin contact, holding position, essential equipment and supplies, discharge criteria, follow-up frequency, indicators and measurement, and medical personnel requirements. ${ }^{6}$

Various studies have emphasized the importance of understanding the barriers to and enablers of KMC at 3 levels, namely, with respect to healthcare providers, caregivers, and facilities, for its successful launch and implementation. ${ }^{7-9)}$ The International Network on KMC suggested proper training for clinicians, adherence to protocols, and the creation of a welcoming environment for families as key elements for effective implementation of KMC.7) In a multicountry study, including $\mathrm{KMC}$ in the preservice curriculum and service training and providing training to other healthcare administrators were facilitating factors for implementing $\mathrm{KMC}{ }^{8}{ }^{8}$ In a qualitative study, the respondents highlighted the importance of ensuring availability of equipment, supplies, facilities, modified patient ward, and quality of services, as well as proper training as critical prerequisites.") Bonding, social support, sufficient mother-infant bonding time, medical concerns regarding the health of the mother or the newborn, availability of KMC and social context were identified as main themes affecting the interaction between families and KMC intervention. ${ }^{10)}$

Based on a descriptive survey performed in Korea, the major barriers to practicing $\mathrm{KMC}$ by nurses were infant safety concerns and the workload of nurses, with $61.1 \%$ of the nurses in 67 neonatal intensive care units adopting KMC. ${ }^{11,12)}$ The respondents $(31.3 \%)$ who had practiced KMC were more knowledgeable and positive in their outlook towards KMC. ${ }^{12)}$ The availability of educational programs and development of KMC practice guidelines are recommended. ${ }^{11)}$

A recent qualitative study assessing knowledge and perception regarding KMC among healthcare providers in 2 district hospitals in Indonesia that the healthcare providers believed that low birthweight infants in incubators could not be treated with KMC and that KMC could be practiced only if a special gown was used to hold the baby. The author attributed this perception to a lack of formal training in KMC, which led to misunderstandings, although the healthcare providers had sufficient knowledge about KMC. However, the results of this study should be interpreted with caution. First, KMC was based on skin-to-skin contact between the mother and preterm baby, and the healthrelated knowledge could have been insufficient. Second, since 1997, the Indonesian Society for Perinatology has been training healthcare providers through workshops in 17 cities within 16 provinces; by the end of 2013, 73 KMC courses were offered to 2,190 medical professionals. ${ }^{13)}$ In 2009 , the Indonesian Ministry of Health provided 3 batches of regional healthcare providers with KMC training courses including training materials, maintenance of records and checklists, and other tools for health personnel. ${ }^{13)}$ However, knowledge regarding KMC was mostly gained through observation, the respondents' own experience, or from pediatricians, indicating the interruption in accessing national $\mathrm{KMC}$ training in Indonesia. Third, various aspects concerning the barriers to and enablers of $\mathrm{KMC}$ at the 3 aforementioned levels must be considered in the implementation of KMC. However, this study included only training aspects tailored to healthcare providers.

In conclusion, KMC should be applied and adopted nationally

Corresponding author: Soon Min Lee, MD. Department of Pediatrics, Gangnam Severance Hospital, Yonsei University College of Medicine, 211 Eonju-ro, Gangnam-gu, Seoul 06351, Korea 
as a premature infant care strategy. Well-trained motivated healthcare providers, required KMC guidelines for facilities, and the establishment of communities of KMC participants are necessary for the successful implementation of KMC. By analyzing the barriers to and building solutions based on national situations, KMC should be integrated into the healthcare system, ultimately improving neonatal outcomes.

\section{Conflicts of interest}

No potential conflict of interest relevant to this article was reported.

See the article "Knowledge and perceptions of kangaroo mother care among health providers: a qualitative study" via https://doi.org/10.3345/cep.2018.06506.

\section{References}

1. World Health Organization. WHO recommendations on interventions to improve preterm birth outcomes. Geneva (Switzerland): World Health Organization, 2015.

2. World Health Organization. Every Newborn: an action plan to end preventable deaths [Internet]. Geneva (Switzerland): World Health Organization; 2014. Available from: https://www.who.int/publications/i/ item/every-newborn-an-action-plan-to-end-preventable-deaths.

3. Conde-Agudelo A, Diaz-Rossello JL. Kangaroo mother care to reduce morbidity and mortality in low birthweight infants. Cochrane Database Syst Rev 2016;2016(8):CD002771.

4. Lim JH, Shin JE, Lee SM, Eun HS, Park MS, Park KI, et al. Efficacy and safety of kangaroo mother care in preterm infants in Korea. Korean J Perinatol 2015;26:305-11.
5. Boundy EO, Dastjerdi R, Spiegelman D, Fawzi WW, Missmer SA, Lieberman E, et al. Kangaroo mother care and neonatal outcomes: a meta-analysis. Pediatrics 2016;137:e20152238.

6. Chan GJ, Valsangkar B, Kajeepeta S, Boundy EO, Wall S. What is kangaroo mother care? Systematic review of the literature. J Glob Health 2016;6:010701.

7. Tamburlini G, Villegas J, Bergh AM. Report on an international workshop on kangaroo mother care: lessons learned and a vision for the future. BMC Pregnancy Childbirth 2018;18:170.

8. Bergh AM, Kerber K, Abwao S, de-Graft Johnson J, Aliganyira P, Davy K, et al. Implementing facility-based kangaroo mother care services: lessons from a multi-country study in Africa. BMC Health Serv Res 2014;14:293.

9. Jamali QZ, Shah R, Shahid F, Fatima A, Khalsa S, Spacek J, et al. Barriers and enablers for practicing kangaroo mother care $(\mathrm{KMC})$ in rural Sindh, Pakistan. PLoS One 2019;14:e0213225.

10. Chan G, Bergelson I, Smith ER, Skotnes T, Wall S. Barriers and enablers of kangaroo mother care implementation from a health systems perspective: a systematic review. Health Policy Plan 2017;32:1466-75.

11. Kim HY, Jang EK, Lee JH, Lee E, Oh S, Jo KS. National survey of kangaroo care practice, barriers, knowledge, and belief. J Korean Clin Nurs Res 2017;23:211-21.

12. Jeong SK, Kim TL. Perception and barriers to kangaroo-mother care among neonatal intensive care unit nurses. Child Health Nurs Res 2016;22:299-308

13. Bergh AM, de Graft-Johnson Joseph, Khadka N, Om'Iniabohs A, Udani $\mathrm{R}$, Pratomo H, et al. The three waves in implementation of facility-based kangaroo mother care: a multi-country case study from Asia. BMC Int Health Hum Rights 2016;16:4. https://doi.org/10.1186/s12914-0160080-4.

How to cite this article: Lee SM. Barriers to and enablers of kangaroo mother care. Clin Exp Pediatr 2020;63:431-2. https:// doi.org/10.3345/kjp.2019.01039. 